\title{
Affective flexibility without perceptual awareness
}

Philipp Homan ${ }^{1}$, H. Lee Lau ${ }^{1}$, Ifat Levy², Candace M. Raio ${ }^{3}$, Dominik R. Bach ${ }^{4,5}$, David Carmel $^{6,7^{*}}$, Daniela Schiller ${ }^{*}$

${ }^{1}$ Department of Psychiatry, Department of Neuroscience, and Friedman Brain Institute, Icahn School of Medicine at Mount Sinai 1470 Madison Ave, NY, NY 10029, USA

${ }^{2}$ Departments of Comparative Medicine, Neuroscience and Psychology

Yale University, New Haven, CT, USA

${ }^{3}$ Department of Psychology, New York University

6 Washington Place, NY, NY 10003, USA

${ }^{4}$ Clinical Psychiatry Research

Department of Psychiatry, Psychotherapy, and Psychosomatics

University of Zurich, Zurich, Switzerland

${ }^{5}$ Wellcome Centre for Human Neuroimaging, 12 Queen Square, London WC1N 3BG, UK

${ }^{6}$ School of Psychology, Victoria University of Wellington,

Easterfield Building, Kelburn Parade, Wellington 6012, New Zealand

${ }^{7}$ Psychology Department, University of Edinburgh, 7 George Square, Edinburgh EH8 9JZ, UK

*These authors contributed equally; address correspondence to daniela.schiller@mssm.edu or david.carmel@vuw.ac.nz 


\section{$1 \quad$ Abstract}

2 In an ever-changing environment, survival depends on learning which stimuli represent threat,

3 and also on updating such associations when circumstances shift. Humans can acquire

4 physiological responses to threat-associated stimuli even when they are unaware of them, but the

5 role of awareness in updating threat contingencies remains unknown. This complex process -

6 generating novel responses while simultaneously suppressing learned ones - relies on distinct

7 neural mechanisms from initial learning, and has only been shown with awareness. Can it occur

8 unconsciously? Here we show that it can. Participants underwent classical threat conditioning to

9 visual stimuli that were suppressed from their awareness. One of two images was paired with an

10 electric shock; halfway through the experiment, contingencies were reversed and the shock was

11 paired with the other image. We found that physiological responses reflected changes in

12 stimulus-threat pairings independently of stimulus awareness, demonstrating the sophistication

13 of unconscious affective flexibility. 


\section{Introduction}

16 Flexible responses to environmental threats are essential for adaptive behavior. Cues that predict

17 threat constantly change - new threats may arise while old ones cease to pose a risk. When

18 consciously perceiving such cues, we are able to flexibly update and shift threat responses from

19 one cue to another (1-3). But can we update our reaction to stimuli that predict danger when we

20 are not aware of them? It is known that threat-conditioned stimuli that are perceived without

21 awareness can still elicit defensive physiological reactions (4-7), and that new threat associations

22 can be formed through classical conditioning even without any awareness of the conditioned

23 stimuli (8-10). Updating threat associations when contingencies change, however, is an entirely

24 different matter: it involves a complex process of creating novel responses while simultaneously

25 suppressing acquired ones. To date, such updating has only been shown in humans who were

26 aware of the stimuli (2), and in animals under conditions where stimuli were fully available for

27 perceptual processing (11); these studies have shown, furthermore, that the neural substrates of

28 threat updating differ from those of the initial learning. It is thus unknown whether the

29 sophisticated re-evaluation involved in such affective flexibility requires awareness, or can be

30 accomplished without it. Here we show that it can, and furthermore, that stimulus awareness

31 does not seem to play a substantial role in such affective flexibility.

32 To examine this, we employed the reversal paradigm, a laboratory model that requires

33 flexible updating of threat contingencies (2). In an initial acquisition phase, participants

34 encounter two conditioned stimuli (CSs) and learn that only one of them predicts an electric

35 shock. Halfway through the experiment, with no warning, these contingencies flip, initiating the

36 reversal phase: Participants must flexibly learn that the formerly safe CS now predicts the shock

37 and that the old one no longer does. To assess learning, participants' physiological arousal is

38 recorded throughout the experiment, typically (and here) by measuring their skin conductance 
responses. Appropriate response reversal requires a sophisticated form of updating, in that one must learn to respond to a cue that now predicts threat while simultaneously inhibiting responses to the previously threatening cue that is now safe.

To see whether reversal of conditioned threat requires awareness, we had a large group of

43 participants $(N=86)$ undergo reversal learning with the CSs suppressed from awareness by

44 continuous flash suppression (CFS), a technique commonly used to examine unconscious

45 perception $(10,12-14)$ : The CSs were visual images presented monocularly, while the other eye was shown a high-contrast, dynamic image (the CFS mask) at the corresponding retinal location

47 (See Figure 1 for a description of the design and procedure).

CFS can suppress images from awareness for several seconds. However, it is also known that its effectiveness may vary across trials and individuals, and the suppressed stimulus may "break through" the suppression (15). Over the last decade, a growing body of work has raised concerns that the standard approach - removing from analysis data (participants and trials) in which

52 breakthrough had occurred - may bias the findings (16, 17; See Supplementary Methods for

53 further details of these issues.) Here, we adopt a number of methodological approaches to ensure 54 our results are robust to these potential concerns.

55 Specifically, we remove no data and instead incorporate individual levels of reported 56 stimulus awareness, as well as response patterns that might reflect residual awareness, into a

57 regression model accounting for physiological responses. The model also adjusts for baseline 58 anxiety (which has been previously shown to correlate with unconscious learning; (10)).

59 Additionally, we use a Bayesian approach to establish that a model in which participants were 60 updating their learning provides a better account for the findings than a model in which they

61 were simply (and independently of the stimulus) predicting the probability of a shock on the next 62 trial (18). Finally, in order to verify that our procedure is able to induce reversal learning when 
63 participants are aware of the stimuli (so that if no learning or updating were found under CFS,

64 we could rule out the possibility this may be simply due to an ineffective procedure) we ran a no-

65 CFS group $(N=12)$, in which participants also viewed the CSs monocularly (as the CFS group

$66 \mathrm{did})$, but were aware of them as no CFS masks were presented to their other eye.

67 We hypothesized that physiological responses to threat can be flexibly reversed without

68 perceptual awareness. We find that reversal indeed occurs independently of CS awareness, and

69 that there is strong evidence for the reversal of threat learning even in its complete absence.

\section{Results}

\section{Overall assessment of physiological reversal learning}

72 To assess the physiological arousal evoked by CSs, we used a model-based approach (19) to

73 estimate the amplitude of anticipatory sudomotor nerve activity (SNA) from skin conductance

74 data recorded during stimulus presentation. A variational Bayes approximation was employed to

75 invert a forward model that describes how hidden SNA translates into observable SCRs (see

76 Materials and Methods). Previous work has shown that this approach is more sensitive than

77 conventional SCR peak-to-peak analysis (19-21). Figure 2A shows the time course of evoked

78 SNA to Spiders A and B, separately for the CFS and no-CFS groups. In both groups, responses

79 to Spider A relative to Spider B were larger during the acquisition phase and smaller during the

80 reversal phase. To quantify the magnitude of physiological reversal learning, we calculated a

81 reversal learning index for each participant (see Materials and Methods). The reversal learning

82 index was positive and significantly greater than zero for both the CFS and no-CFS groups

83 (Figure 2B). A linear mixed model (see Materials and Methods for details) revealed a significant

84 interaction of stage and spider in both groups (CFS: $\beta=0.27, t(2935)=4.23, P=<0.001$; no-

85 CFS: $\beta=1.23, t(2935)=7.29, P=<0.001)$. Note that a significant interaction is formally 
equivalent to a significant reversal learning index. On its own, however, it simply reveals a

87 difference in the comparative magnitude of responses to the two CSs across the two halves of the experiment; follow-up tests show that this difference is indeed due to reversal: Spider A evoked greater responses than Spider B in the acquisition phase (CFS: $t(341.9)=3.0, P=0.003$; no-

90 CFS: $t(201.1)=4.6, P<0.001)$ and the pattern was reversed in the reversal phase (CFS: $t$

$91(341.9)=2.8, P=0.005$; no-CFS: $t(341.9)=3.6, P=0.0003)$. These results indicate that

92 reversal learning was evident in both groups. Although Figure 2 shows that it was more

93 pronounced in the no-CFS group, we note that this difference is not straightforwardly

94 interpretable because the no-CFS group (a control, intended to rule out an ineffective

95 manipulation if no effect was found for the CFS group) was substantially smaller; furthermore,

96 as addressed in detail below, suppression from awareness was very heterogenous in the CFS

97 group.

98 As previous work has found a negative association between anxiety and threat acquisition

99 with and without awareness (10), we also calculated correlations between the CFS group's

100 baseline anxiety measures (STAIT, STAIS, FSQ) and the reversal learning index. Overall,

101 reversal learning decreased significantly with increasing levels of state and trait anxiety, and to a

102 lesser but non-significant extent for spider phobia (Figure 2C).

103 Reversal learning and perceptual awareness

104 The CFS manipulation reduced awareness of the CSs; as expected, however, it was differentially 105 effective in doing so across participants, precluding an overall conclusion that all learning under

106 CFS happened non-consciously. The CFS group showed significantly lower accuracy in response

107 to the "which seen?" question $(\mathrm{M}=0.46, \mathrm{SD}=0.29)$ compared to the no-CFS group $(\mathrm{M}=0.86$,

$108 \mathrm{SD}=0.16 ; t(22.77)=-7.24, P<0.001)$, and accuracy in the CFS group was not significantly 
different from the $50 \%$ random-response level $(t(85)=-1.21, P=0.229)$. The CFS group also

110 showed lower confidence $(\mathrm{M}=1.73, \mathrm{SD}=0.65)$ than the no-CFS group $(\mathrm{M}=2.83, \mathrm{SD}=0.08 ; t$

$111(95.38)=-15.05, P<0.001)$.

112 However, group differences in accuracy and confidence, and even random-level response

113 accuracy, are not sufficient to establish an absence of perceptual awareness in the CFS group.

114 Notably, average confidence of correct responses in this group was low but significantly greater

115 than the minimum value of $1(t(77)=10.79, P<0.001)$, suggesting that at least some

116 participants were aware of some of the CSs; learning might thus have arisen from a subset of

117 trials and/or participants where such awareness occurred. To address this, we quantified CS

118 awareness by calculating an awareness index for each participant, ranging in possible values

119 from 0 for no awareness to 1 for full awareness (see Materials and Methods). Although the

120 awareness index of the CFS group $(\mathrm{M}=0.28, \mathrm{SD}=0.34)$ was significantly lower than the no-

121 CFS group's $(\mathrm{M}=0.92, \mathrm{SD}=0.18 ; t(23.93)=-10.19, P<0.001)$, it was still significantly

122 higher than zero $(t(85)=7.59, P<0.001)$.

123 Therefore, in order to test our main hypothesis that the reversal of acquired threat responses

124 can be achieved without perceptual awareness, we characterized the quantitative relation

125 between the level of awareness and the magnitude of reversal learning in the CFS group. To

126 control for possible artifacts of regression to the mean (see Supplementary Methods), we

127 followed the recommendation (16) to first examine the correlation between two independent

128 estimates of the awareness index, one calculated from even-numbered trials, the other from odd-

129 numbered trials. Because noise at the measurement level might occasionally yield extreme (i.e.,

130 very low or very high) awareness index scores, an association of such randomly-extreme scores

131 with reversal learning (specifically, low awareness with intact learning) could be an artifact.

132 However, it is highly unlikely that across participants, random noise would yield consistent (and 
133 similarly extreme) measurements in separate estimates. Due to regression to the mean, if random

134 extreme values occur in one of the two estimates, they are less likely to occur in the other,

135 resulting in a considerable attenuation of any correlation between the two. We found, however,

136 that the two measures were strongly correlated $(r(84)=0.96, P<0.001$; Figure $3 \mathrm{~A})$;

137 participants' awareness level in one set of trials was overwhelmingly predictive of their

138 awareness in the other set, confirming their reliability as estimates of awareness.

139 Next, we examined the association between the awareness index and the reversal learning

140 index, using values of both indices obtained separately from even (Figure 3B) and odd (Figure

$1413 \mathrm{C}$ ) trials. As the color-coding of Figure 3 shows, the relation between individual participants'

142 awareness and their reversal learning was highly consistent across these separate measurements.

143 In light of this, we pooled the data from all trials and regressed the reversal learning index on the

144 perceptual awareness index (Figure 3D). The parameter of interest was the intercept, i.e., the

145 magnitude of reversal learning at zero perceptual awareness. The intercept was positive and

146 significantly different from zero. Furthermore, the awareness index regressor did not contribute

147 significantly to prediction of reversal learning; importantly, this finding was even stronger in

148 models that accounted for STAIT scores and a binary factor indicating whether participants were

149 tracking the stimuli with their responses (see Materials and Methods; Figure 3E and Table 1).

\section{Comparing learning and expectation-based accounts}

151 Well-controlled lab-based conditioning procedures require strict constraints that preclude

152 complete randomization of the number and order of different CSs; this comes with a cost:

153 participants are able to develop expectations with above-chance validity, based on the sequence

154 of trials so far, about the likelihood of a shock on any upcoming trial (18). Even without any

155 awareness of the CSs, a participant should have been able to distinguish two types of trials: 
156 reinforced (with shock) and non-reinforced (no-shock). In a study with two CSs and a 100\%

157 reinforcement rate like ours, such expectations would correspond to an anticipated pattern of

158 alternating trial-types (shock/no-shock or vice versa), with an increase in shock anticipation after

159 every no-shock trial. The question, therefore, was whether the physiological responses we had

160 measured might simply reflect participants' pattern-based anticipation of shock, rather than

161 learning of the contingencies associated with the CSs.

162 To answer this question, we used a Bayesian approach to compare the probability of our

163 findings being accounted for by a classic Rescorla-Wagner learning model (22) and a trial-

164 sequence model. We hypothesized that successful threat reversal without perceptual awareness

165 should be better explained by the Rescorla-Wagner learning model, whereas simple pattern-

166 based expectation would be better explained by the trial-sequence learning model. We used

167 maximum likelihood estimation to assess the log likelihood and calculate the Bayesian

168 Information Criterion (BIC) of each model (See Materials and Methods for details of each model

169 and calculation of the BIC). A smaller BIC indicates a better model, and BIC values can thus be

170 compared by calculating the difference between them and interpreting the resulting $\Delta \mathrm{BIC}$ as

171 providing evidence against the higher BIC. The Rescorla-Wagner model (BIC: 562.1)

172 outperformed the pattern-based expectation model (BIC: 584.9$)$, with the difference $(\Delta \mathrm{BIC}$ :

17322.9 ) greater than 10, suggesting that the evidence against the trial switch model is very strong

174 (23). Repeating this comparison for just the participants with zero mean awareness confirmed the

175 lower BIC for the Rescorla-Wagner model (BIC: 114.3) compared to the pattern-based

176 expectation model (BIC: 125.7), with the difference again greater than $10(\Delta \mathrm{BIC}: 11.3$; see also

177 Figure S2). This model comparison provides convincing evidence that a classical Rescorla-

178 Wagner learning model explains our findings better than an alternative expectation-based model. 


\section{Discussion}

180 These results indicate that participants updated their defensive physiological responses

181 independently of their awareness of threat-related cues. Previous studies have shown that new

182 threat associations can be formed without perceptual awareness of the conditioned stimuli $(5,9-$

183 10). However, until now it was unknown whether the far more complex process of threat reversal

184 - shifting reactions from a stimulus that no longer predicts danger to one that now does - can be

185 accomplished without awareness. The independence of reversal learning from perceptual

186 awareness, reported here, suggests that separate processes underlie affective flexibility and

187 conscious processing (24). Conversely, the negative correlation between reversal learning and

188 anxiety suggests that the various impairments caused by anxiety are not limited to the systems

189 underlying conscious processes.

190 Previous studies have pointed out the limitations of using accuracy and confidence measures

191 to assess perceptual awareness, and suggested remedies including the calculation of

192 metacognitive sensitivity measures (25), Bayesian statistics (26), or parametric variation of the

193 experimental manipulation (27). The present study addresses an issue not covered in previous

194 discussions, by showing that a trial-wise analysis may reveal hints for incomplete suppression

195 that analyses relying on average measures might easily miss. Future studies that rely on forced-

196 choice questions for awareness assessment should thus examine response patterns across trials in

197 addition to collecting aggregate measures.

198 Notably, a previous study (10) that used CFS to investigate acquisition of threat responses

199 without awareness of the stimuli found that such acquisition can occur, but is rapidly forgotten.

200 The present study again showed that such acquisition can occur (and, additionally, be reversed),

201 but did not find the same rapid forgetting. The reasons for this are unclear, but we speculate that

202 the difference may be due to specific aspects of the stimuli, design and procedure: our use of 
203 pictures of spiders (rather than the faces used in the previous study) and a 100\% (rather than

$20450 \%$ ) reinforcement protocol may have altered the temporal characteristics of acquisition.

205 Similarly, the temporal profile of reversal may change if the stimuli and reinforcement regime

206 are different.

207 The present results add to a growing body of findings distinguishing functions that do and do

208 not require awareness. Such distinctions are important in guiding research into the neural

209 mechanisms of conscious and non-conscious processing. Previous research hints at the

210 mechanism underlying the non-conscious affective flexibility reported here, although it remains

211 to be elucidated: The ability to reverse conditioned responses depends on the integrity of

212 circuitry spanning several neural regions, particularly the ventromedial prefrontal cortex

213 (vmPFC) and its connections with the amygdala (1) where threat associations are formed (28).

214 Consistent with this, it is known that patients with anxiety disorders often show rigid and

215 inflexible threat responses in conjunction with prefrontal cortex dysfunction $(29,30)$.

216 Indeed, the real-life settings that people with anxiety disorders find challenging often require

217 the updating and shifting of threat responses. Deficits in affective flexibility may thus explain the

218 threat learning and extinction deficits seen in such disorders (31): Compared to healthy controls,

219 patients are less able to distinguish between safe and unsafe stimuli in threat learning (when it is

220 adaptive to do so), and distinguish between them to a greater extent during extinction (when it is

221 non-adaptive). Threat learning without perceptual awareness is also negatively correlated with

222 baseline state anxiety in healthy participants (10). Our new finding that baseline anxiety is

223 negatively correlated with affective flexibility suggests a potential use for reversal learning as a

224 model paradigm for investigating how anxiety modulates various processes in a variety of

225 disorders, including, for example, posttraumatic stress disorder, in which there is an impairment

226 of threat inhibition (32). 


\section{Methods}

\section{Participants}

229 Ninety-eight healthy participants (mean age $=29.97$; range 18-65) were assigned to one of the

230 two groups: reversal learning with CFS (CFS group; $N=86,48$ female) or without CFS (no-CFS

231 group; $N=12,5$ female). Assignment was random until each group reached a size of 12;

232 subsequent participants were assigned to the CFS group. Measures of trait and state anxiety

233 (Spielberger Trait-State Anxiety Inventory (33); STAIT and STAIS, respectively) and spider

234 phobia (Fear of Spider Questionnaire; FSQ (34)) were taken prior to participation and did not

235 differ between the groups (Table S1). The experiment was approved by the Institutional Review

236 Board of the Icahn School of Medicine at Mount Sinai. All participants provided written

237 informed consent and were financially compensated for their participation.

\section{Experimental procedure}

239 Participants viewed the stimuli monocularly, through a mirror stereoscope (StereoAids,

240 Australia) placed at a distance of $45 \mathrm{~cm}$ from a 17-inch Dell monitor. The CSs (schematic low-

241 contrast images of spiders), presented to the left eye only, were suppressed from awareness in the

242 CFS group: while the left eye saw them, the right eye was presented with "Mondrians" - arrays

243 of high contrast, multi-colored, randomly generated rectangles alternating at $10 \mathrm{~Hz}$. Both the CSs

244 and the CFS masks were flanked by identical textured black and white bars, to facilitate stable

245 ocular vergence. The no-CFS group viewed identical CSs (also presented monocularly), but with

246 no Mondrians presented to the other eye.

247 The experiment consisted of 16 acquisition trials followed by 16 reversal trials. One of two

248 spider images was presented on each trial. The spider images were schematic and had similar

249 low-level features. During acquisition, spider A always terminated with a shock and spider B 
never did. Reversal occurred halfway through the experiment: spider B now terminated with a

251 shock and spider A did not. The spider stimuli were presented for $6 \mathrm{~s}$ each in pseudorandomized

252 order. One of four possible trial orders was used for each participant. Orders were generated by

253 imposing specific constraints on the trial order, such that the first trial was always reinforced and

254 no more than two of the same trial type ever occurred consecutively.

255 Trial order and spider identity were counterbalanced across participants. To assess the

256 effectiveness of the awareness manipulation (35), $1 \mathrm{~s}$ after the offset of every CS participants

257 were shown the question "Which seen?" ( 1 = flower, 2 = spider; notably, flowers were never

258 shown, meaning the question addressed detection rather than discrimination as it could be

259 answered correctly even with a brief glimpse). This was followed by the question "How

260 confident?" ( 1 = guess to 3 = sure; participants were instructed to indicate how confident they

261 were of the flower/spider answer they had just given). Both questions were presented binocularly

262 (1.5 - $2 \mathrm{~s}$ each, during which responses had to be given by pressing number keys on a standard

263 keyboard). The second question was followed by an 8 to $10 \mathrm{~s}$ inter-trial interval.

\section{Psychophysiological stimulation and measurement}

265 Mild electric shocks were delivered using a Grass Medical Instruments SD9 stimulator and

266 stimulating bar electrode attached to the participant's right wrist. Shocks (200 ms; 50 pulse/s)

267 were delivered at a level determined individually by each participant as "uncomfortable but not

268 painful" (maximum of $60 \mathrm{~V}$ ), during a work-up procedure prior to the experiment.

269 Skin conductance responses (SCR) were measured with $\mathrm{Ag}$-AgCl electrodes, filled with

270 standard isotonic $\mathrm{NaCl}$ electrolyte gel, and attached to the middle phalanges of the second and

271 third fingers of the left hand. SCR signals were sampled continuously at a rate of $200 \mathrm{~Hz}$, 
272 amplified and recorded with a MP150 BIOPAC Systems skin conductance module connected to

273 a PC.

\section{Analysis of physiological responses}

\section{Model-based analysis}

276 We estimated SNA from SCR data with a model-based variational Bayes approximation (19),

277 inverting a forward model that describes how (hidden) SNA translates into (observable) SCR. A

278 unit increase in SNA corresponds to an increase in SCR of 1 micro Siemens. The model assumes

279 that the observed SCR can be decomposed into different components including anticipation,

280 evocation, and spontaneous fluctuations, each of which are generated by bursts of SNA driven by

281 changes in sympathetic arousal. The generative (forward) model thus describes how sympathetic

282 arousal, the physiological measure that is taken as an index of the psychological process of

283 threat, translates into sudomotor nerve bursts which then generate the observable SCR (19).

284 Using Bayesian inference, the forward model can then be reversed in order to estimate the most

285 likely underlying SNA given the observed SCR:

$$
p(\theta \mid y)=\frac{p(\theta) p(y \mid \theta)}{p(y)}
$$

287 where the most likely parameter vector $\Theta$ (corresponding to the SNA) given the observed

288 outcome $y$ (corresponding to the SCR) is given by the prior estimate of $\Theta$ weighted by the

289 likelihood of $y$ given $\Theta$. Solving this equation involves integration over the model evidence $p(y)$

290 which is analytically hard to compute (and possibly intractable). This can be resolved by

291 replacing this integration problem by an optimization problem, which can be approximated with

292 Variational Bayes procedures (36), where the log of the model evidence can be framed as the 
293 sum of the Kullback-Leibler divergence and the Free Energy. By maximizing the Free Energy

294 the Kullback-Leibler divergence is minimized, and a lower bound to the log model evidence can

295 be derived iteratively.

296 The SNA estimates were computed using previously developed software package PsPM (19)

297 implemented in MATLAB R2016b (The Mathworks Inc, Natick, MA, USA). The statistical

298 analyses were conducted with the R software (37) (R version 3.4.2 (2017-09-28)) and the

299 libraries lme4 (38) and lsmeans (39). Welch's t-tests were used instead of two sample t-tests

300 when groups had unequal variances.

\section{$301 \quad$ Reversal Learning Index}

302 An estimate of SNA was obtained for each trial. We expected Spider A to evoke greater SNA

303 than Spider B during the acquisition phase, and Spider B to evoke greater SNA than Spider A

304 during the reversal phase. The strength of reversal learning can thus be quantified by calculating,

305 separately for the acquisition and reversal phases, the difference between the average SNA

306 evoked by each spider. To quantify the degree of reversal (which is formally equivalent to the

307 interaction of phase and stimulus), the reversal learning index was calculated by subtracting the

308 difference between mean SNAs evoked by each spider during reversal from the difference during

309 acquisition (the larger the index, the greater the magnitude of reversal learning):

$$
\begin{aligned}
\text { Reversal learning index } & =\Delta \text { Acquisition }-\Delta \text { Reversal } \\
\Delta \text { Acquisition } & =[\text { mean }(\text { Spider } \mathrm{A})-\text { mean }(\text { Spider } \mathrm{B})]_{\text {Acquisition }} \\
\Delta \text { Reversal } & =[\text { mean }(\text { Spider } \mathrm{A})-\text { mean }(\text { Spider B })]_{\text {Reversal }}
\end{aligned}
$$

311 To formally test for group differences in the strength of reversal learning, we computed a

312 linear mixed model using the lme4 library in R. We used the skin conductance response 
313 (converted to a model-based measure of sudomotor nerve activity, SNA) as the dependent

314 variable and entered group (CFS, no-CFS), stage (acquisition, reversal), and spider (spider A,

315 spider B) as well as a continuous variable for trial (to account for habituation) as predictors. The

316 random structure of the model included an intercept and slopes for stage and spider.

\section{Assessments of perceptual awareness}

\section{Perceptual awareness index}

319 To characterize participants' reported awareness of CSs, each trial was assigned a perceptual

320 awareness score, defined by a combination of detection and confidence responses: Correct

321 answers with a confidence rating of 1 (guess) and incorrect answers irrespective of confidence

322 were assigned an awareness score of 0 ; correct answers with a confidence rating of 2 (medium)

323 were assigned a score of 0.5 , and correct answers with a confidence rating of 3 (high) were

324 assigned an awareness score of 1. A perceptual awareness index was calculated for each

325 participant by averaging awareness scores across all trials.

\section{Stimulus-response association patterns ("tracking")}

327 We also assessed response patterns across trials, to see whether participants were able to track

328 stimuli with their responses, accurately discriminating the images despite not being able to label

329 them. We plotted individual trial-by-trial responses to the question "Which seen?", overlaid on

330 the trial-by-trial presentation of spiders (spider A, spider B; Figure S1A). We then calculated the

331 number of consecutive "hits", defined as the number of consecutive trials where these two time-

332 courses were either identical or consistently in opposition, suggesting that there was a possible

333 association between the stimulus and the response during those trials. The probability of such

334 consecutive hits occurring by chance alone can be derived as follows: 
336 left, $i$ the number of consecutive hits already observed; the chance of observing $k$ consecutive

337 hits for the remaining $n$ trials can then be formulated as a recursive problem:

$$
f_{p, k}(i, n)=p f_{p, k}(i+1, n-1)+(1-p) f_{p, k}(0, n-1)
$$

339 which can be solved analytically with dynamic programming or recursion. Trivially, $f p, k(k, n)=$

3401 for $n \geq 0$ since $k$ consecutive hits have already been observed, and $f_{p}, k(i, n)=0$ for $k-i>n$

341 since there are not enough trials left to observe $k$ consecutive hits.

342 For example, assuming we want to know how likely it is to observe $k=8$ consecutive hits

343 within $n=32$ trials given $p=0.5$, i.e., $f 0.5,8(0,32)$, we find that this yields a probability of

3440.050.

345 Alternatively, the probability can be derived by simulation for all possible numbers of

346 consecutive hits within 32 trials (i.e., from 1 to 31 ). For each possible number, we thus also

347 simulated $10^{5}$ draws of a binomial distribution and calculated the average probability of that

348 number of hits being consecutive. As can be seen in Figure S1B, the result for 8 consecutive hits

349 (0.04991) was very close to the analytical solution. Fifteen participants showed evidence of

350 tracking the spiders or the shocks with their responses (8 or more consecutive hits); notably, 3 of

351 these participants appeared to have a perceptual awareness index of zero. We thus adjusted our

352 subsequent analysis with an additional binary covariate, indicating whether participants did or

353 did not show 8 or more consecutive hits. 


\section{Comparing learning and expectation-based models}

355 The Rescorla-Wagner model (22) describes how the prediction for each trial is updated

356 according to a prediction error and learning rate:

$$
\begin{aligned}
V_{n+1}\left(x_{n}\right) & =V_{n}\left(x_{n}\right)+\alpha \delta_{n} \\
\delta_{n} & =r_{n}-V_{n}\left(x_{n}\right),
\end{aligned}
$$

where $x_{n}$ is the conditioned stimulus on trial $n$ (Spider A or Spider B), and $\delta_{n}$ is the punishment prediction error that measures the difference between the expected and the actual shock $(r n)$ on trial $n$. The learning rate $\alpha$ for the value update is a constant free parameter. The value for the CS not observed on trial $n$ remains unchanged. To derive the best fits for the Rescorla-Wagner model, we assumed that $V_{0}=0.5$, reflecting the assumption that getting a shock or not was equally likely for the first trial.

For the alternative trial-sequence learning model, we assumed that a participant expecting a

$$
\begin{aligned}
V_{n+1}^{\prime} & =V_{n}^{\prime}+\alpha^{\prime} \delta_{n}^{\prime} \\
\delta_{n}^{\prime} & =r_{n}^{\prime}-V_{n}^{\prime} \\
\tau_{n} & =\left|\left(r_{n-1}^{\prime}-1\right)\right|,
\end{aligned}
$$

where $V_{n+1}$ is the expected trial type switch at trial $n+1$ (if $V_{n+1}^{\prime}$ is larger than 0.5 , a trial switch corresponds to the difference between the actual trial type switch for trial $n\left(r^{\prime} n\right.$; coded as one for

371 a trial type switch and zero for an equal trial type) and the expectation for trial $\mathrm{n}$. A changing 
373 the preceding trial type was one. To map these expectations onto expected values, we assumed

374 that

$$
V_{n+1}= \begin{cases}V_{n+1}^{\prime} \cdot \tau_{n}+\left(1-V_{n+1}^{\prime}\right)\left(1-\tau_{n}\right), & \text { if } V^{\prime}>0.5 \\ V_{n+1}^{\prime}, & \text { otherwise }\end{cases}
$$

376 where the expected value for trial $n+1$ was calculated according to whether a trial type switch

377 was expected $\left(V^{\prime}>0.5\right)$ or not.

378 We performed a formal model comparison between the conventional Rescorla-Wagner

379 model and the trial switch model for our data set (Figure S2), using maximum likelihood

380 estimation and non-linear optimization (implemented with the fmincon function in MATLAB

381 R2016b (The Mathworks Inc, Natick, MA, USA). Using the log likelihood, we calculated the

382 Bayesian Information Criterion (BIC) to compare the two models as follows:

$$
\mathrm{BIC}=\log (n) k-2 \cdot \log (\hat{L}),
$$

384 where $n$ is the number of data points, $k$ is the number of regressors, and $k$ is the maximized

385 value of the likelihood function. 


\section{References}

388 1. Morris JS, Dolan RJ (2004) Dissociable amygdala and orbitofrontal responses during 389 reversal fear conditioning. Neuroimage 22(1):372-80.

390 2. Schiller D, et al. (2008) From fear to safety and back: Reversal of fear in the 391 human brain. Journal of Neuroscience 28(45):11517-25.

392 3. Fleming SM, Dolan RJ, Frith CD (2012) Metacognition: computation, biology and function. Philosophical Transactions of the Royal Society of London. Series B, Biological Sciences 367(1594):1280-6.

4. Critchley HD, Mathias CJ, Dolan RJ (2002) Fear conditioning in humans: the influence of awareness and autonomic arousal on functional neuroanatomy. Neuron 33(4):653-63.

5. Morris JS, Ohman A, Dolan RJ (1998) Conscious and unconscious emotional learning in the human amygdala. Nature 393(6684):467-70.

6. Ohman A, Soares JJ (1994) "unconscious anxiety": phobic responses to masked stimuli.

401 7. Whalen PJ, et al. (1998) Masked presentations of emotional facial expressions modulate amygdala activity without explicit knowledge. Journal of Neuroscience 18(1):411-8.

8. Katkin ES, Wiens S, Ohman A (2001) Nonconscious fear conditioning, visceral perception, and the development of gut feelings. Psychol Sci 12(5):366-70.

9. Manns JR, Clark RE, Squire LR (2002) Standard delay eyeblink classical conditioning is independent of awareness. Journal of Experimental Psychology: Animal Behavior Processes 28(1):32-7.

10. Raio CM, Carmel D, Carrasco M, Phelps EA (2012) Nonconscious fear is quickly acquired but swiftly forgotten. Current Biology 22(12):R477-9. 
410 11. Izquiredo A, Brigman JL, Radke AK, Rudebeck PH, Holmes A (217) The neural basis of

411 reversal learning: An updated perspective. Neuroscience 345:12-26.

412 12. Tsuchiya N, Koch C (2005) Continuous flash suppression reduces negative afterimages.

$413 \quad$ Nature Neuroscience 8(8):1096-101.

414 13. Stein T, Hebart MN, Sterzer P (2011) Breaking continuous flash suppression: A new

415 measure of unconscious processing during interocular suppression? Frontiers in Human

$416 \quad$ Neuroscience 5:167.

417 14. Carmel D, Arcaro M, Kastner S, Hasson U (2010) How to create and use binocular rivalry.

$418 \quad$ J Vis $\operatorname{Exp}(45)$.

419 15. Gayet S, Stein T (2017) Between-subject variability in the breaking continuous flash

420 suppression paradigm: Potential causes, consequences, and solutions. Frontiers in

$421 \quad$ Psychology 8:437.

422 16. Shanks DR (2016) Regressive research: The pitfalls of post hoc data selection in the study

423 of unconscious mental processes. Psychonomic Bulletin \& Review 24:752-775.

424 17. Stein T, Sterzer P (2014) Unconscious processing under interocular suppression: getting

425 the right measure. Frontiers in Psychology, 5, 387.

426 18. Wiens S, Katkin ES, Ohman A (2003) Effects of trial order and differential conditioning

427 on acquisition of differential shock expectancy and skin conductance conditioning to

428 masked stimuli. Psychophysiology 40(6):989-97.

429 19. Bach DR, Daunizeau J, Friston KJ, Dolan RJ (2010) Dynamic causal modelling of

430 anticipatory skin conductance responses. Biological Psychology 85(1):163-70.

431 20. Bach DR (2014) A head-to-head comparison of scralyze and ledalab, two model-based

432 methods for skin conductance analysis. Biological Psychology 103:63-8. 
433 21. Staib M, Castegnetti G, Bach DR (2015) Optimising a model-based approach to inferring

434 fear learning from skin conductance responses. Journal of Neuroscience Methods 255:131-

4358.

436 22. Rescorla R, Wagner A (1972) A theory of Pavlovian conditioning: Variations in the

437 effectiveness of reinforcement and nonreinforcement, eds. Black A, Prokasy W. (Appleton-

438 Century-Crofts, New York), pp. 64-99.

439 23. Raftery AE (1995) Bayesian model selection in social research. Sociological methodology

$440 \quad$ pp. $111-163$.

441 24. Lau H, Rosenthal D (2011) Empirical support for higher-order theories of conscious awareness. Trends in Cognitive Sciences 15(8):365 - 373.

443 25. Fleming SM, Lau HC (2014) How to measure metacognition. Frontiers in Human $444 \quad$ Neuroscience 8:443.

445 26. Dienes Z (2015) How Bayesian statistics are needed to determine whether mental states 446 are unconscious, ed. Overgaard M. (Oxford University Press, Oxford), pp. 199-221.

447 27. Schmidt T (2015) Invisible stimuli, implicit thresholds: Why invisibility judgments cannot 448 be interpreted in isolation. Adv Cogn Psychol 11(2):31-41.

449 28. Roy M, Shohamy D, Wager TD (2012) Ventromedial prefrontal-subcortical systems and 450 the generation of affective meaning. Trends in Cognitive Science 16(3):147-56.

451 29. Ressler KJ, Mayberg HS (2007) Targeting abnormal neural circuits in mood and anxiety 452 disorders: from the laboratory to the clinic. Nature Neuroscience 10(9):1116-24.

453 30. Rauch SL, Shin LM, Phelps EA (2006) Neurocircuitry models of posttraumatic stress 454 disorder and extinction: human neuroimaging research-past, present, and future. Biological 455 Psychiatry 60(4):376-82. 
456 31. Duits P, et al. (2015) Updated meta-analysis of classical fear conditioning in the anxiety 457 disorders. Depression and Anxiety 32(4):239-253.

458 32. Jovanovic T, Norrholm SD (2011) Neural mechanisms of impaired fear inhibition in 459 posttraumatic stress disorder. Frontiers in Behavioral Neuroscience 5:44.

460 33. Spielberger CD (1983) State-trait anxiety inventory for adults. PsycTESTS Dataset.

461 34. Szymanski J, O’Donohue W (1995) Fear of spiders questionnaire. PsycTESTS Dataset.

462 35. Merikle PM, Smilek D, Eastwood JD (2001) Perception without awareness: perspectives 463 from cognitive psychology. Cognition 79(1-2):115-34.

464 36. Friston K, Mattout J, Trujillo-Barreto N, Ashburner J, Penny W (2006) Variational free 465 energy and the laplace approximation. NeuroImage 34(1):220-234.

466 37. R Core Team (2016) R: A Language and Environment for Statistical Computing (R 467 Foundation for Statistical Computing, Vienna, Austria).

468 38. Bates DM (2005) Fitting linear mixed models in R. R News 5:27-30.

469 39. Lenth RV (2016) Least-squares means: The R package lsmeans. Journal of Statistical $470 \quad$ Software 69(1):1-33. 


\section{Acknowledgments}

472 We thank Patrik Vuilleumier who created and shared the spider stimuli. This work was

473 supported in part through the computational resources and staff expertise provided by Scientific

474 Computing at the Icahn School of Medicine at Mount Sinai. Funding was provided by NIMH

475 grant MH105515 and a Klingenstein-Simons Fellowship Award in the Neurosciences to D.S.;

476 ERC Advanced Grant XSPECT-DLV-692739 to D.C. (Co-I); and Swiss National Science

477 Foundation grant SNF 161077 to P.H.

\section{Author Contributions}

479 PH carried out the computational modeling and statistical analysis, interpreted the results, and

480 drafted the manuscript. HL prepared materials, collected the data and critically revised the

481 manuscript. IL contributed to the conception of the study, the computational modeling, the

482 interpretation of the results, and critically revised the manuscript. CMR contributed to the

483 interpretation of the results and critically revised the manuscript. DRB contributed to the

484 computational modeling and critically revised the manuscript. DS conceived, designed and

485 coordinated the study, contributed to data analysis and interpretation, and critically revised the

486 manuscript. DC contributed to the conception of the study, data analysis and interpretation of

487 results, and drafted the manuscript in its final form. All authors gave final approval for

488 publication.

\section{Conflict of Interests}

490 All authors declare no conflicts of interest with regard to the current study. 


\section{Figures and Tables}

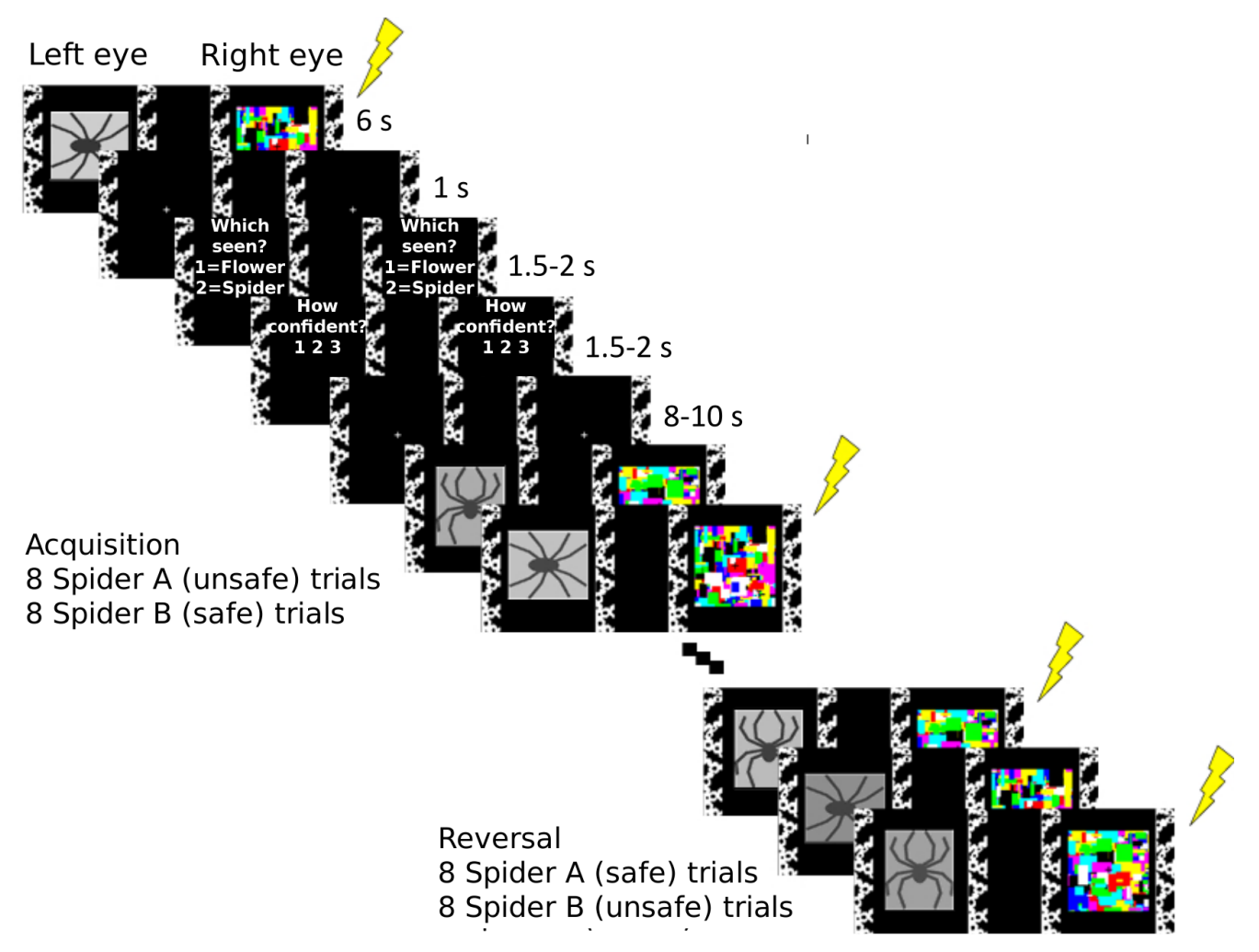

Figure 1: Schematic description of experimental design and procedure. In each trial of the acquisition phase, participants were presented with one of two stimuli (schematic pictures of spiders, presented monocularly for $6 \mathrm{sec}$ and suppressed from awareness by a CFS mask shown to the other eye). One image (spider A) always terminated with a mild electric shock to the wrist, whereas the other (spider B) never did. Halfway through the experiment, with no warning, the contingencies flipped and the reversal phase began: the formerly safe stimulus (spider B) now predicted the shock, and the old threat-associated one (spider A) was now safe. Each spider was shown 8 times in each phase. Trial order was pseudorandomized (see Materials and Methods) and spider identity (A and B) was counterbalanced across participants. To assess the success of the awareness manipulation, participants answered the questions "Which seen?" (1=flower, $2=$ spider) and "How confident?" (1=guess to $3=$ sure), presented binocularly ( 1.5 - $2 \mathrm{~s}$ each), beginning $1 \mathrm{~s}$ after the offset of every CS, and followed by an 8-10 s inter-trial interval (the questions are only shown here for the first depicted trial, but were repeated in all trials). Participants who underwent the same procedure without CFS were shown identical CSs, but the 507 CFS mask was absent. 
A

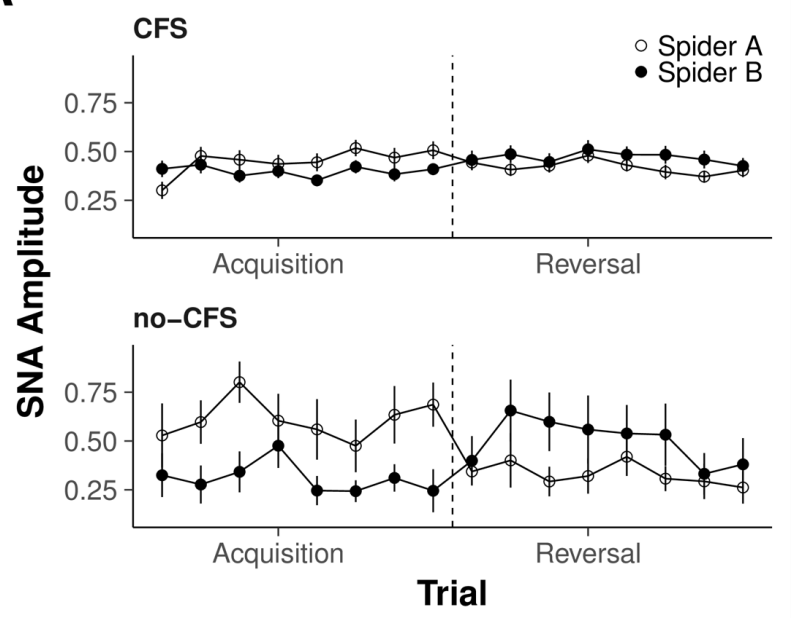

B

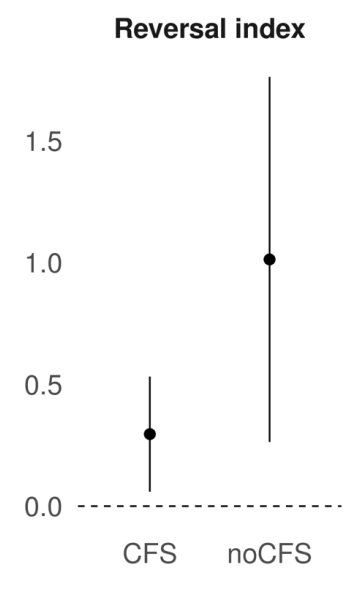

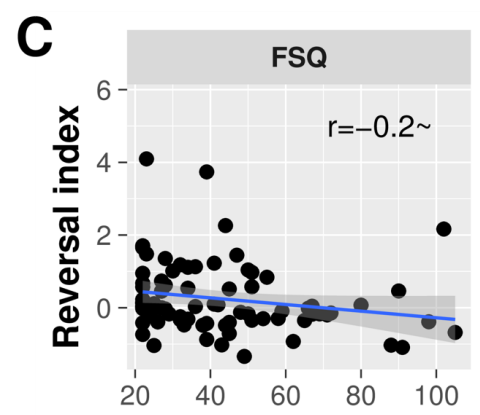

Figure 2: Physiological reversal learning. A. Time courses reveal reversal of threat responses with and without continuous flash suppression. Data points represent trial-wise mean responses to spider A (the CS+ during acquisition) and spider B (the CS- during acquisition). Both groups showed reversal learning, as indicated by greater responses to Spider A during the acquisition phase and greater responses to Spider B during the reversal phase. Error bars represent standard errors. B. Mean reversal learning index for each group. Error bars represent $95 \%$ confidence intervals, indicating that the interaction of stage and stimulus and thus the magnitude of reversal learning in both groups was significantly greater than zero. $\mathbf{C}$. Heightened anxiety is associated with impaired reversal learning under CFS. A negative correlation between baseline anxiety measures and the strength of threat reversal learning is evident for state and trait anxiety. Blue lines show linear fits of each score to the reversal index, and ribbons around lines indicate bootstrapped $95 \%$ confidence intervals around the estimate. Abbreviations: STAIS/STAIT, state/trait anxiety subscale of the Spielberger State-Trait Anxiety Inventory; FSQ, Fear of Spider Questionnaire, $\sim, P<.1 ;{ }^{*}, P<.05$. 


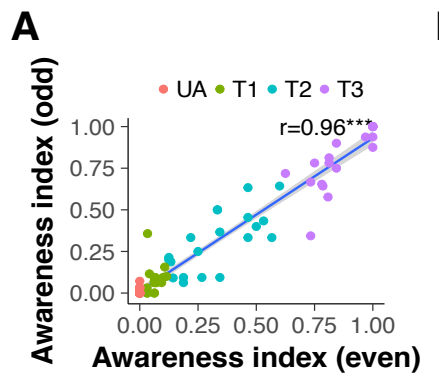

D

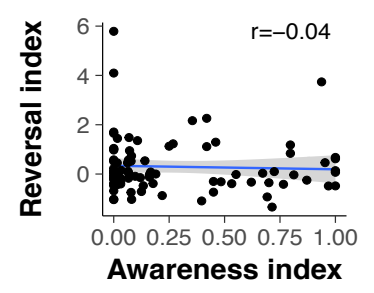

B C
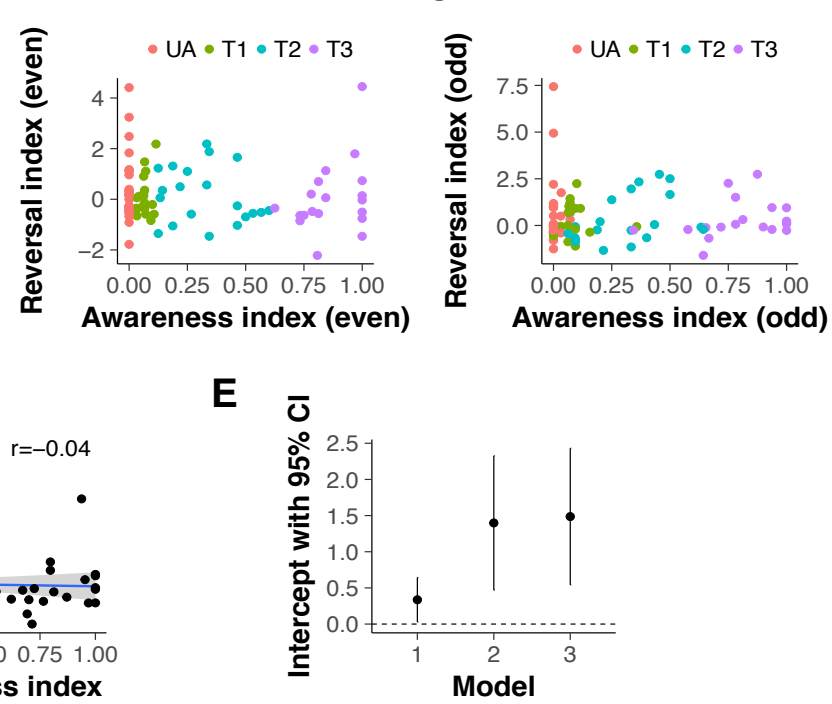

Figure 3: Characterizing the relation between perceptual awareness and reversal learning in the CFS group. A. Correlation between the awareness index of even and odd-numbered trials. Each data point represents an individual participant. The strong positive correlation between these independent measures of awareness demonstrates that individual participants' awareness ratings - even those with extreme values of zero or one - are unlikely to be due to measurement noise. For illustrative purposes, the color scheme marks all participants with an awareness index of 0 in even trials in red (UA, unaware, $N=27$ ) and classifies the rest of the CFS group in 3 tertiles (T1-T3). Note that some data points overlap. B. Reversal learning plotted against perceptual awareness for individual participants, for data obtained from even-numbered trials. The color scheme is the same as in Panel A. C. Reversal learning plotted against perceptual awareness for individual participants, for data obtained from odd-numbered trials. Individual participants are marked with the same color as in the previous panels; the overall distribution of participants is highly similar across panels. D. Reversal learning as a function of perceptual awareness in the CFS group, using data pooled from all trials. The intercept, indicating the magnitude of reversal learning in the absence of awareness, is positive and significantly different from zero. E. Reversal Index intercepts and their $\mathbf{9 5 \%}$ confidence intervals in a series of regression models. Model 1 depicts the intercept (the value of the reversal index when the awareness index equals zero) shown in Panel D. Model 2 shows the intercept when the regression model includes STAIT scores in addition to the perceptual awareness index. Model 3 regresses the reversal index onto the perceptual awareness index, STAIT and tracking scores. (Excluding the potential outlier in the top left corner of panel D weakens significance of the intercept in model $1, P=0.07$; the intercepts of model 2 and 3 remain significant after removal of this outlier). Blue lines show linear fits, and ribbons around lines indicate bootstrapped $95 \%$ confidence intervals around the estimate. 
554 Table 1: Regression coefficients for all awareness index models. Reversal learning was the 555 dependent variable in all models. Model 1 included an intercept and the perceptual awareness 556 index; model 2 additionally included STAIT scores; model 3 additionally included STAIT and 557 tracking scores. 